\title{
Aerodynamic Optimization of Airborne Radome for Maritime Patrol Radar
}

\author{
M R Shankar, A C Niranjanappa, B Dattaguru
}

\begin{abstract}
Airborne Early Warning (AEW) systems are deployed for getting surveillance information on airborne enemy targets. Electromagnetic sensors such as Radars are integrated on airborne platforms for collecting such information. Maritime Patrol Radar (MPR) is used for surveillance of sea surface for various types of ships and low flying aircraft. The antenna of MPR is belly mounted on typical turbo prop aircraft and protected from environment with a cover called Radome. Airborne radomes are electromagnetically transparent. The radome installation introduces additional drag which will reduce the range of the aircraft. To minimise the drag due to installation of radome, the profile has to be stream-lined or optimised with CFD analysis for certain operational points of aircraft flight. Design of radome is multidisciplinary effort involving Aerodynamics, Structures and Electromagnetic disciplines. In this study, aerodynamic optimization of a radome for a given antenna size is carried out using a combination Genetic Algorithm (GA) and traditional optimisation methods to find the Utopia point for further investigation on Multidisciplinary Design Optimization (MDO) of radome. This is necessary to progress on optimisation with other disciplines like Structures and Electromagnetics (EM)
\end{abstract}

Keywords: Airborne Radomes, Optimization, Aerodynamics, Sandwich Structure

\section{INTRODUCTION}

Airborne Early Warning (AEW) systems are used for airborne surveillance to get advance information on the incoming enemy fighters or targets. RADAR is electromagnetic system which is integrated on suitable airborne platform such as executive jets or turbo-prop aircraft for carrying out airborne surveillance and early warning. Maritime Patrol Radar (MPR) is such a radar which provides surveillance information on the targets which are flying low above the sea surface or enemy ships sailing on the seas. As part of MPR system, an antenna is installed below the fuselage to transmit / receive ElectroMagnetic (EM) signals. A typical installation of MPR Radome is illustrated in Fig. 1. To protect an antenna from environmental conditions like, rain, sandstorm, hail etc., a cover is required. This cover is termed radome and is electromagnetically transparent.

Revised Manuscript Received on February 05, 2020.

* Correspondence Author

M R Shankar*, Scientist F, CABS, DRDO, Bengaluru 560037 and Research Scholar Jain (deemed-to-be-University), Bengaluru 562112 , India Email: shankar_mr@yahoo.com

Dr A C Niranjanappa, Outstanding Scientist, CABS, DRDO, India 560037 Email: niranjanappa@cabs.drdo.in

Dr B Dattaguru, Professor IIAEM, Jain (deemed-to-be-University), Bengaluru 562 112, India Email: datgurb@gmail.com

(C) The Authors. Published by Blue Eyes Intelligence Engineering and Sciences Publication (BEIESP). This is an open access article under the CC BY-NC-ND license (http://creativecommons.org/licenses/by-nc-nd/4.0/)
It has to offer least resistance to electromagnetic signals transmitted/received and at the same time structurally strong enough to withstand air loads and other environmental effects [1].

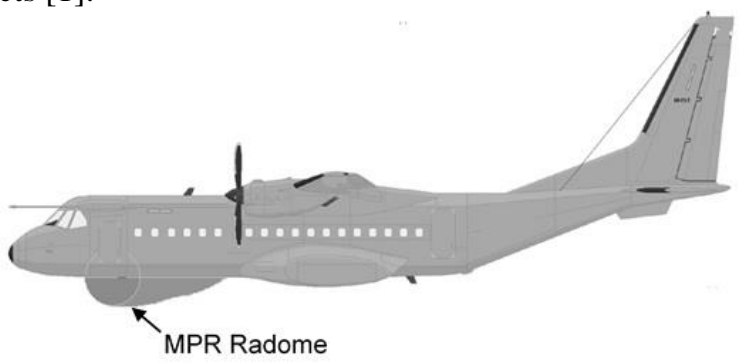

Figure 1. Installation of Maritime Patrol Radar Antenna and Radome

In this paper, an approach for the optimization of the shape the airborne radome from aerodynamic performance is presented as a part of the larger study on Multidisciplinary Design Optimization (MDO) of the radome.

\section{REVIEW OF LITERATURE}

Effect of drag on the shape and location of airborne radome is studied by Girgosian P et al [2]. It is evident that the location and the length to height ratio of radome have large effect on the radome drag. Other parameters that have significant effect are radome's wetted area, width and subtended angle at the tail portion. Effect on radome sizing and location on the drag have been studied for various configurations and reported.

Satellite Communication (SATCOM) radomes used for land, shipboard, or airborne applications should protect the antenna system from environmental conditions like rain, winds, ice, saline environment etc. Also the physical impact of the sea waves has to be accounted for radomes in maritime environment. A multi objective optimization approach with electrical and mechanical design considering cost also is suggested by $\mathrm{O}$ Russo et al [3]. Authors have used a sequential approach in selection of radome materials, analyses for electrical and mechanical properties, multiobjective optimization for composite layup design, experimental verification on samples and full scale testing of the final products. In the present work, a mathematical model is evolved which can integrate various disciplines and carry out multi-disciplinary optimization.

Toivanen, J. et al [4], have studied optimization of an airfoil shape involving aerodynamics and electromagnetics. The drag coefficient and strength of backscattered radiation (RCS) of an airfoil are minimized in a multi-objective optimization problem setup. 
Drag computation is based on Euler equations. Makinen, R. A. E. et al [5] studied the above problem by employing Navier Stokes equations solver for CFD and same Helmholtz equation for CEM. Renuka. A. et al [6] have discussed the design aspects of dielectric airborne radomes. It is highlighted that the design of radome is generally driven by aerodynamic considerations. Slotted waveguide antenna pattern with and without the radome is analysed using a software based on physical optics method. The output of the software is the estimate of bore sight error with respect to scan angles.

In multi-objective optimization, there are several methods to attach priority to the objectives. Marler. et al [7] discussed about two broad approaches for assignment of weights for the objectives, namely, ranking and rating approach. The importance and role of weights in MDO are brought out and it is informed that the weights represent the change in preference function of objectives.

Lee. K. W. et al [8] proposed a simple design equation for A-sandwich radome design for aircrafts, missiles as well as fixed ground installations. Radomes are proposed as thin or multiple half-wavelengths $(\lambda / 2)$. Radomes with good EM transmission properties are with the thickness of $\lambda / 20$. This radome will not be able to sustain harsh environmental conditions and is structurally weak. Half-wavelength radomes are sturdy but have very narrow band characteristics. Hence a sandwich panel with two skins (one on either side) of $\lambda / 20$ thickness and a core of $\lambda / 2$ thickness has good mechanical strength as well as good transmission characteristics. This paper arrives at the empirical formulae to arrive at the skin thickness (d) and core thickness based on the operational frequency. These compare well with some experimental results.

\section{AIRBORNE RADOMES}

RADOME (RADar Dome) is a cover that gives environmental protection to the Radar Antenna. Radomes can be of monolithic or sandwich construction according to the type of construction. Since the radomes have to be electromagnetically transparent, dielectric materials such as Glass fibre and Aramid fibre are used for construction of radomes. According to dielectric wall construction, different styles of radomes are defined, which are namely thin walled, half wavelength, A-Sandwich, B-Sandwich and C-Sandwich and multi-layered [1]. Fig. 2 shows typical radome styles. A-Sandwich and C-Sandwich radomes are widely used in aircraft. Since softer core is present on either side in BSandwich it is not generally used. Radomes are also classified as Constant Thickness (CT) and Variable Thickness (VT) radomes.



Figure 2. Airborne Radome Classification [1]
For the design of radomes, typically E-Glass or Quartz is used for composite skins (layers). Quartz offers lowest EM signal loss in Glass fibres and hence it is often preferred. Non-metallic Honeycombs and many types of foams are used as cores in Sandwich constructions.

\section{RADOME DESIGN- MULTIDISCIPLINARY EFFORT}

Any external installation of a structure on the fuselage of an aircraft offers resistance to air flow during its flight thereby increasing the drag. The drag has an undesired effect of reducing aircraft range and its speed. As radome is a necessity to protect the antenna from environment, it is imperative that the effect of its installation, i.e., drag, has to be reduced as much as possible.

Radome design is a multidisciplinary design effort involving structural, aerodynamic and electromagnetic disciplines. The design requirements for each of these disciplines could be contradictory in nature. For the structures, the radome needs to be thin and as small as possible to be least in weight. For aerodynamics, to have least drag, the structure has to be streamlined and slender. For least losses in terms of electromagnetics, the radome has to be thinner and flatter. Hence radome design is dependent on the materials used (strength characteristics), its size (length, width and height), radome thickness, type of construction (monolithic or sandwich) and shape. Each of these parameters or variables affect

(a) Weight of the radome

(b) External disturbance it will cause in flight i.e., drag and

(c) Electromagnetic losses such as Radar Transmission Efficiency (RTE) and Insertion Phase Delay (IPD)

Given the choice of material, i.e., Glass Fibre and Honeycomb, the design variables in the design of radome are its shape (function of its profile), size and thickness.

\section{Optimization Problem Statement}

The optimization problem of radome can be expressed as
Minimise $F=f\left(J_{1}(x), J_{2}(x), J_{3}(x)\right)$
Subject to $a \leq x \leq b$

$x=L, B, H, S(L, W, H), t$ where $F$ is the objective function to be minimised and is itself a function of $J_{1}$ (drag), $J_{2}$ (weight) and $J_{3}$ (EM loss). $\boldsymbol{x}$ is vector of design variables which are length $L$, width $B$, height $H$, thickness $t$, and profile $S$ of radome. Profile $\mathrm{S}$ is a function of $L, W, H$. Lower and upper bounds for the design variables are defined by the vectors $\boldsymbol{a}$ and $\boldsymbol{b}$. The limits imposed on the design variables are called the side constraints in the optimization problem.

This is a MDO problem with more than one objective to be optimised. It involves multidisciplinary analyses i.e., Structural, Aerodynamics and Electromagnetics which need to be integrated to interact with each other. Each of these disciplines is required to evaluate the objective functions and the combination of three objectives from three disciplines is to be minimised. The optimization function is obtained from Eqn. 1 as below 


$$
\text { Minimise } F=\left(w_{1} J_{1}(x)+w_{2} J_{2}(x)+w_{3} J_{3}(x)\right)
$$

where $w_{i}$ are weights assigned to each disciplinary objective. Various schemes for assigning weights have been reviewed by Marler [7]. Normalization of the objectives is discussed and methods to normalize the function before assigning weights are elaborated.

A Utopia point is a point in the design space where all the objectives are simultaneously at their optimum; however, this is not achievable in reality. Knowledge of the Utopia point is a requirement for most of the schemes where weights are to be assigned to the objectives. The optimum points when each of these objectives is considered in isolation is to be determined to arrive at the weights and scheme for implementation of weights to be attached to each of the objectives while handling Multi-objective problem. In this study the design is presented where the optimum (minimum) objective is the drag.

\section{RADOME SHAPE AND ITS AERODYNAMIC EFFECT}

Radomes are generally axisymmetric about the longitudinal axis of the aircraft. Three-Dimensional (3D) shape of the radome is derived from a $2 \mathrm{D}$ spline which is defined on $\mathrm{XY}$ plane of aircraft. Fig. 3 shows typical 2D spline which defines the radome cross section. When this $2 \mathrm{D}$ spline is revolved $180^{\circ}$ about longitudinal axis, radome's $3 \mathrm{D}$ shape is obtained. Radome shape determines the drag for a given size of radome and for a given flight condition. With the 3D shape of the radome determined, thickness can be added to radome. Different layers of laminates of the radome and the core thickness can then be defined as part the overall thickness of radome. For the present study, Constant thickness (CT) radome with A-Sandwich construction is considered.

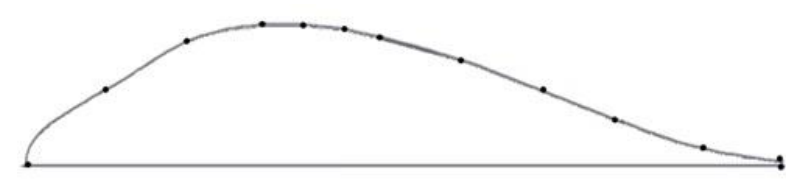

Figure 3. Typical Radome Cross Section

The radome has to have a minimum volume at least equal to that of the antenna it needs to house. An antenna is installed inside the radome and it is rotated $360^{\circ}$ about its axis in the azimuth for sending and receiving EM signals. Hence a minimum swept volume created by rotating a flat plate about an axis perpendicular to longitudinal axis, i.e., a cylinder with a minimum diameter and height needs to be housed in the radome. This cylinder defines the lower limits for the height and width of the radome at the location where the antenna has to be installed. Fig. 4 shows the radome 3D shape and minimum swept volume required for installation of antenna inside the radome.



Figure 4. Typical Radome 3D shape

\section{AERODYNAMIC OPTIMIZATION}

\section{A. Problem Statement}

Aerodynamic optimization is carried out for a chosen operational point of aircraft, i.e, for the aircraft flying at 0.3 Mach $(103 \mathrm{~m} / \mathrm{s})$ air speed and at 2438m (8000ft) altitude. Problem statement of aerodynamic optimization problem can be stated as

$$
\begin{aligned}
& \text { Minimise } \boldsymbol{f}=\boldsymbol{J}(\boldsymbol{x}) \\
& \text { subject to } \boldsymbol{a} \leq \boldsymbol{x} \leq \boldsymbol{b}
\end{aligned}
$$

where $J(x)$ is Drag due to radome and $\boldsymbol{x}$ is vector of design variables (as given in Table 1). Aim of the optimization study is to arrive at a radome shape for which the drag experienced is minimum, at the selected operational point of the maritime aircraft. This has to be achieved by estimating drag for various radome profiles and mathematically choosing the shape for which the drag is near minimum.

For studying radomes of different lengths and contours, the profile of radome is varied by varying the $\mathbf{X}$ and $\mathbf{Y}$ coordinates of various control points or Design Variables (DV) (see Fig. 5) within lower and upper bounds (as in Table 1).

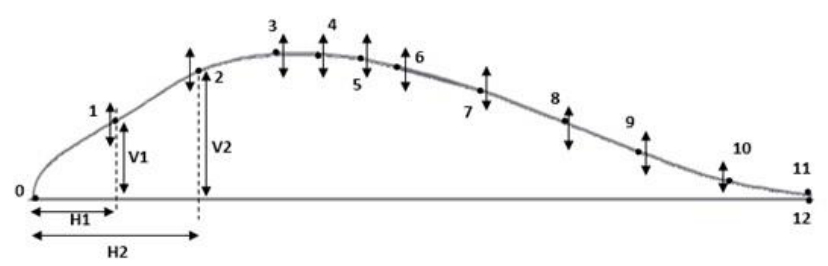

Figure 5. Variation of control points of radome

In Fig. 5, points 1 to 11 are the control points which determine the cross section of the radome under study. Point 0 is fixed at 0,0 (origin) and the point 12 is fixed at L, 0 where $\mathrm{L}$ is the length of the radome. All the $\mathrm{X}$ coordinates (i.e., horizontal distance from point 0 ) are varied as a percentage of total length " $\mathrm{L}$ ". The $\mathrm{Y}$ coordinates of each of these control points is varied within the upper and lower bounds (see Table 1). All the dimensions are in "metres". 
Aerodynamic Optimization of Airborne Radome for Maritime Patrol Radar

Table 1. Design variables and their bounds

\begin{tabular}{|c|c|c|c|c|c|c|}
\hline $\begin{array}{c}\text { Variable } \\
\text { Description }\end{array}$ & $\begin{array}{c}\text { Design } \\
\text { Variable }\end{array}$ & Unit & Initial value & $\begin{array}{c}\text { Lower } \\
\text { bound }\end{array}$ & $\begin{array}{c}\text { Upper } \\
\text { bound }\end{array}$ & Step Size \\
\hline Total Length & $\mathrm{L}$ & $\mathrm{m} *$ & 1.8 & 1.5 & 3.0 & 0.075 \\
\hline Stn 1 Length & $\mathrm{H} 1$ & $\%^{* *}$ & 0.12 & 0.10 & 0.15 & 0.0025 \\
\hline Stn 2 Length & $\mathrm{H} 2$ & $\%$ & 0.22 & 0.20 & 0.25 & 0.0025 \\
\hline Stn 3 Length & $\mathrm{H} 3$ & $\%$ & 0.32 & 0.30 & 0.35 & 0.0025 \\
\hline Stn 4 Length & $\mathrm{H} 4$ & $\%$ & 0.42 & 0.40 & 0.45 & 0.0025 \\
\hline Stn 5 Length & H5 & $\%$ & 0.52 & 0.50 & 0.55 & 0.0025 \\
\hline Stn 6 Length & H6 & $\%$ & 0.62 & 0.60 & 0.65 & 0.0025 \\
\hline Stn 7 Length & H7 & $\%$ & 0.72 & 0.70 & 0.75 & 0.0025 \\
\hline Stn 8 Length & H8 & $\%$ & 0.82 & 0.80 & 0.85 & 0.0025 \\
\hline Stn 9 Length & H9 & $\%$ & 0.92 & 0.90 & 0.94 & 0.0025 \\
\hline Stn 10 Length & H10 & $\%$ & 0.97 & 0.96 & 0.98 & 0.0025 \\
\hline Stn 11 Length & H11 & $\%$ & 0.995 & 0.99 & 1.0 & 0.0025 \\
\hline Stn 1 Height & V1 & $\mathrm{m}$ & 0.2 & 0.17 & 0.25 & 0.005 \\
\hline Stn 2 Height & V2 & $\mathrm{m}$ & 0.275 & 0.25 & 0.3 & 0.005 \\
\hline Stn 3 Height & V3 & $\mathrm{m}$ & 0.325 & 0.30 & 0.35 & 0.005 \\
\hline Stn 4 Height & V4 & $\mathrm{m}$ & 0.30 & 0.25 & 0.35 & 0.005 \\
\hline Stn 5 Height & V5 & $\mathrm{m}$ & 0.25 & 0.20 & 0.30 & 0.005 \\
\hline Stn 6 Height & $\mathrm{V} 6$ & $\mathrm{~m}$ & 0.20 & 0.15 & 0.25 & 0.005 \\
\hline Stn 7 Height & V7 & $\mathrm{m}$ & 0.20 & 0.15 & 0.25 & 0.005 \\
\hline Stn 8 Height & V8 & $\mathrm{m}$ & 0.175 & 0.15 & 0.20 & 0.005 \\
\hline Stn 9 Height & V9 & $\mathrm{m}$ & 0.125 & 0.10 & 0.15 & 0.005 \\
\hline Stn 10 Height & V10 & $\mathrm{m}$ & 0.055 & 0.01 & 0.1 & 0.005 \\
\hline Stn 11 Height & V11 & $\mathrm{m}$ & 0.029 & 0.008 & 0.05 & 0.005 \\
\hline & & & & & & \\
\hline
\end{tabular}

*meters

** as percentage of current iteration length (L)

\section{B. CFD analysis}

Drag of a chosen radome shape needs to be estimated using CFD simulation in ANSYS. To start with, profile (2D) of radome is modelled with initial values of the control points and a spline is created by joining these points. The 3D shape is then created by revolving the $2 \mathrm{D}$ spline 180 deg about $\mathrm{X}$ axis, as explained in section VI. The control points (design variables) are defined as parameters in ANSYS so that these can be imported into optimization software for defining upper and lower bounds (side constraints). The optimization software will vary the values of the design variables based on the values of the objective (drag) at each of the iterations. The flow domain is modelled and CFD mesh is created with using tetrahedral mesh and prism layers. Parameters of the CFD mesh are given Table 2.

The parameters of CFD mesh are arrived at after a convergence analysis for initial geometry. To capture the boundary layer effect, prism layers are created adjacent to radome wall. CFD mesh and the prism layers are shown in Fig. 6 and Fig. 7 respectively. The initial and boundary conditions are defined for the flow analysis. The mesh gets automatically resized in ANSYS software based on the changed geometry points fed by the optimization software. Some analysis runs had returned zero value of drag since the mesh resizing had failed in those cases.
Table 2. CFD Domain details

\begin{tabular}{|l|l|}
\hline CFD domain size & $3.6 \mathrm{~m}$ upstream \\
& $18 \mathrm{~m}$ downstream \\
& $3.6 \mathrm{~m}$ above \\
& $5.4 \mathrm{~m}$ on both side \\
\hline Number of elements & $6,63,888$ \\
\hline Prism layers & 20 \\
\hline First layer height & $0.02 \mathrm{~mm}$ \\
\hline Growth Rate & 1.19 \\
\hline Average Y + & 2.16139 \\
\hline Boundary conditions & Inlet, outlet, wall and symmetry \\
\hline
\end{tabular}

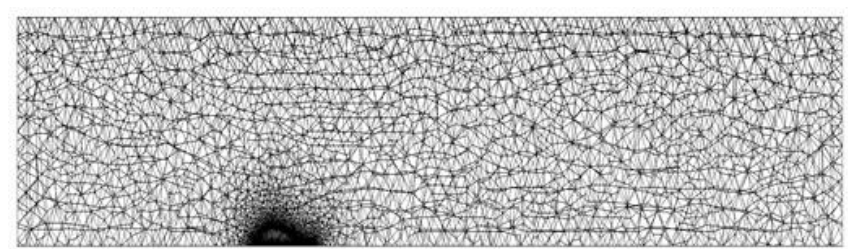

Figure 6. CFD Mesh

CFD run for each geometry is an iterative process and gets converged when the residuals are less than the defined value. Drag value which is the one of the outputs of ANSYS, is linked to optimization software to estimate the objective value of Eqn. 5. Each of the CFD run to evaluate the drag lasts about 15 to 20 minutes. 


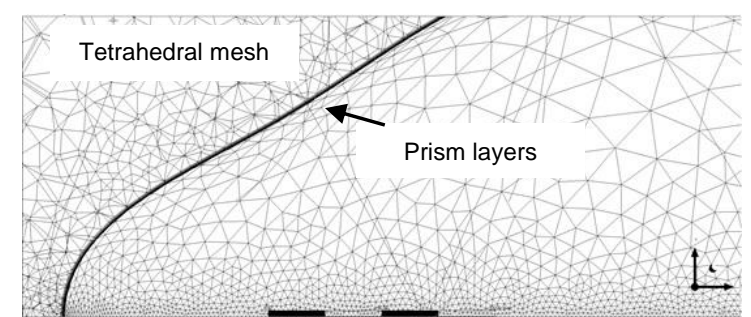

Figure 7. Prism layers

\section{Optimization Process}

The optimization process is carried out by optimization software modeFrontier. For carrying out the optimization, the inputs (design variables) and output (drag) of the CFD software are linked to the optimizer algorithm. The process is briefly explained in Fig. 8. The optimizer creates a design table (step 1), i.e., combination of different values of design variables. The optimiser invokes ANSYS Workbench in batch mode and passes on the initial values of design variables (step 2). These design variables which are defined as parameters in ANSYS are used to create new profile of radome (step 3). ANSYS software then runs flow simulation using Fluent code (step 4) and determines the drag value (step 5) for the current shape of the radome. This drag value is read by modeFrontier to evaluate the objective function (step 6). Based on the value of objective function, new set of values for the design variables are determined (step 8) by the optimiser algorithm and the same is again sent for carrying out CFD simulation. The steps 3 to 8 are repeated in a loop. Exiting the loop (step 7) is based on the stop criterion which explained in following paragraphs.

An adaptive multi strategy optimization algorithm called pilOPT is used to carry out the optimization. Depending on number of design variables, number of objective function or functions, and the time for each iteration, the algorithm adopts design space exploration and optimization strategy such as Response Surface Model based optimization, Genetic algorithms, Evolution strategy, Particle swarm intelligence, simulated annealing, game theory, gradient based methods to find the direction of search and the minima. In general terms, the design space is initially explored with Genetic Algorithm (GA) to fully explore the design space in order to find out the extent of impact of the design variables on the objective function. Gradient based approach is then used to localise and find the optimum value of objective function based on the gradient information of objective function.

The absolute minimum is generally never reached in such a problem and hence the stop criterion is number of iterations. Additionally, the operation loop terminates if the improvement in objective function in successive iterations is less than a prescribed value. In an autonomous mode the algorithm stops further evaluation when the Pareto front does not improve further for multi-objectives problem.

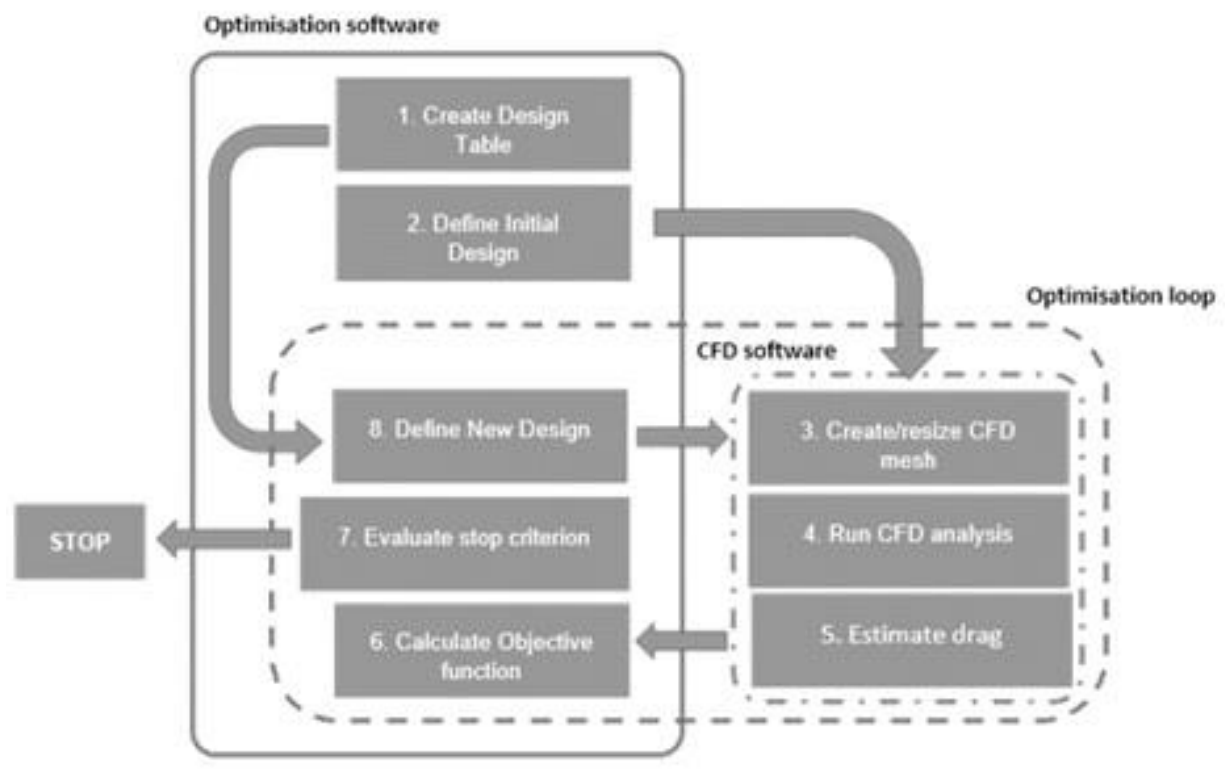

Figure 8. Steps in Optimization Process

The control points are varied within the upper and lower limits so as to explore different shapes of the radome. When the value for adjacent control points are varied within the limits, it ends up in wavy shapes and smooth shapes as well. The objective (drag) is evaluated and the optimiser drives towards the profile having lesser drag values. Some profiles generated by optimizer and evaluated by the CFD software are depicted in Fig. 9. In this it is clear that the optimizer has explored the design space thoroughly and generated different profiles for various lengths of the radome. The natural selection is towards most streamlined profiles which give lower values of drag. Around 800 iterations (profiles) are generated and evaluated by optimizer to arrive at the profile with minimum drag.

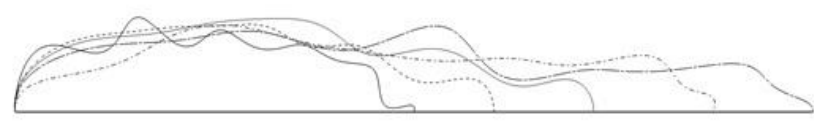

Figure 9. Some of the profiles studied
Published By:

Blue Eyes Intelligence Engineering \& Sciences Publication 


\section{Aerodynamic Optimization of Airborne Radome for Maritime Patrol Radar}

\section{RESULTS AND DISCUSSION}

In the initial stages, the optimiser tries to evaluate designs to the full extent of the design space, i.e., it tries to explore the whole design space which is defined by the limits for all design variables. Based on initial evaluations and the value of objective (drag) of these designs, optimiser tries to localise where possible minimum values could occur. A case of how the designs of different radome profiles are explored for a length of $2.25 \mathrm{~m}$ is shown in Fig. 10.



Figure 10. Optimization progress for $2.25 \mathrm{~m}$ length radome

Initially more designs with varying drag values are evaluated with uneven profiles also being included for design exploration (case of 153N). Later profiles become smoother (case of $57.5 \mathrm{~N}$ ) and get further streamlined (case of $34 \mathrm{~N}$ ). Similar trend is seen in all the radome lengths explored from $1.5 \mathrm{~m}$ to $3.0 \mathrm{~m}$.

Progress of optimisation of all length cases is shown in Fig. 11. Here, up to around 100 iterations or so, radome designs evaluated have drag values from as low as $50 \mathrm{~N}$ to as high as $300 \mathrm{~N}$. As the optimisation progresses, this value narrows down and reduces to less than $50 \mathrm{~N}$ in phased manner and gets restricted to a narrow band. The radome profiles get more streamlined as is evident with lower values of objective values with the progress of optimization. The optimizer process terminates when the reduction in the drag values become lesser and lesser. The optimization process took nearly 36 hours for completion.

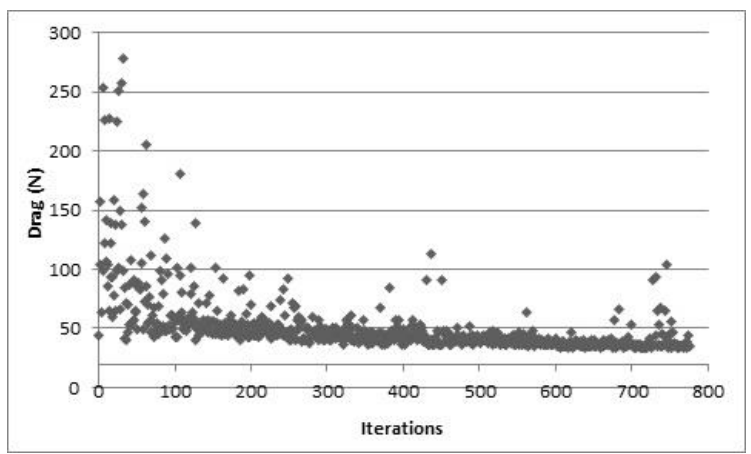

Figure 11. Trend of objective values

Fig. 12 shows the initial profile, the radome profiles which have highest (worst) drag value and the least (best) drag values. Due to unevenness in the profile, the drag is high for this profile. This is one of the designs evaluated initially. Among the designs evaluated, the highest drag value is $279 \mathrm{~N}$ for a radome of $1.5 \mathrm{~m}$ length. The best profile is fully streamlined and hence the drag values drops down to $33.5 \mathrm{~N}$.
Further continuation of optimization does not result in much reduction in this value and hence it can be terminated.



Figure 12. Initial, worst and best radome profiles

Drag value is the sum the of pressure (form) drag and skin friction drag as computed by CFD software. Pressure drag depends on the form of radome and skin friction drag depends on its length, which is again a trade-off. Longer stream lined radomes will have less form drag but skin friction drag will be more. Shorter radomes will have less skin friction due to lower wetted area but have higher form drag due to higher pressure gradients. Optimized radome is shown in Fig. 13. Length of this radome is $2.48 \mathrm{~m}$ and drag value of this radome is $33.5 \mathrm{~N}$.

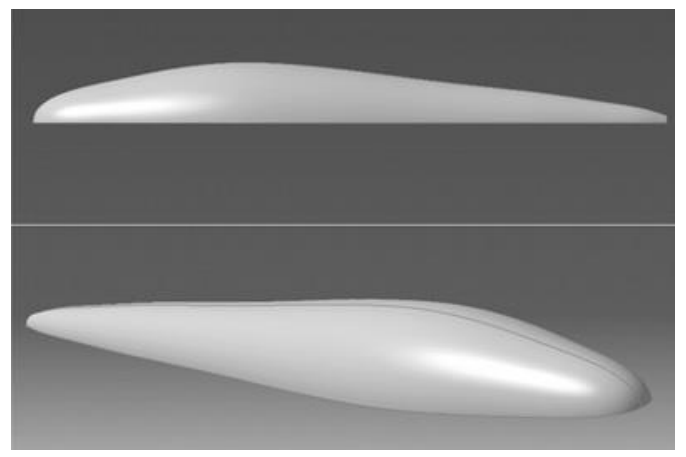

Figure 13. Optimised Radome Shape

\section{CONCLUSIONS}

In this study, aerodynamic optimization is performed using ANSYS and modeFrontier optimization software by varying radome lengths and the profiles while ensuring antenna of known size can be accommodated inside the radome. The results indicate that the sleeker profile (lower $\mathrm{l} / \mathrm{d}$ ratios) provide lesser drag values. The best profile in terms of least drag is established using optimisation algorithms, which is subject to lower and upper bounds for design variables and constraints. Present study considered aerodynamic aspects of the radome design and evaluated best design with respect to this single discipline and Utopia point with respect to aerodynamics is found. The structural and EM aspects of the radome are to be integrated to carry out multi-objective MDO analysis and optimization. The Utopia points with respect to structures and EM also needs to be explored.

\section{ACKNOWLEDGMENT}

Authors acknowledge permission given by Mr. M S Easwaran, Distinguished Scientist and Director, CABS, DRDO for carrying out this study with the software and computing facility at CABS aircraft group. The support provided by colleagues in Aircraft group, CABS is appreciated.

Published By:

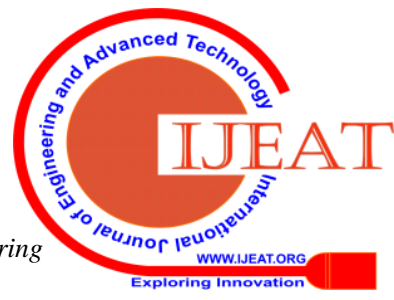




\section{REFERENCES}

1. Military specification: General Specifications for Radomes, MIL-R7705 B, 1975

2. Girgosian P., et al. "Aerodynamic design considerations for aircraft radomes." Presented at the17th Atmospheric conference on Flight Mechanics, held at Portland, USA, 1990

3. Russo O., et al, "State of the Art Materials for KU and KA band Mobile Satellite Antenna Radomes", Telecommunications Ground Segments Workshop, ESA/ESTEC, Noordwijk, Netherlands, 2012

4. Toivanen, J., et al. "Multidisciplinary shape optimization in aerodynamics and electromagnetics using genetic algorithms." Intl J Numerical Methods in Fluids, Vol.28, 1999

5. Makinen, R. A. E., et al. "A genetic algorithm for multi-objective design optimization in aerodynamics and electromagnetics." 4th ECCOMAS Computational Fluid Dynamics Conference, Athens, 7-11 Sept. 1998.

6. Renuka, A. et al, "Computer-aided analysis for tangent Ogive airborne radome using physical optics method," 2005 Asia-Pacific Microwave Conference Proceedings, Suzhou, China, 2005

7. Marler, R., et al. "The weighted sum method for multi-objective optimization: new insights." Structural and multidisciplinary optimization 41.6 (2010): 853-862

8. Lee K W., et al, "An Effective Design Procedure for A-Sandwich Radome", Proceeding of IEEE Antennas and Propagation Society, Toronto, 2010

\section{AUTHORS PROFILE}



Mr. M R Shankar, is PhD scholar in IIAEM, Jain (Deemed to be university), Bengaluru and Scientist F \& Head Aircraft Division in Centre for Airborne Systems (DRDO), Bengaluru, India. He has more the 20 years in experience in aircraft structural modification \& integration, new product development, structural design and optimisation.

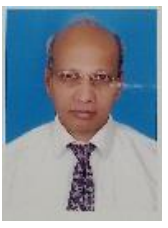

Dr AC Niranjanappa, Outstanding Scientist is Group Director for Aircraft Operations at Centre for Air Borne Systems (DRDO), Bengaluru, India. He is an expert in the field of Advanced Polymer Composites and specialized in the design and development of sandwich radomes for airborne applications. He has been working in the field of Aircraft Structural modifications, integration of Airborne surveillance mission systems and flight test R\&D activities from the last 30 years.

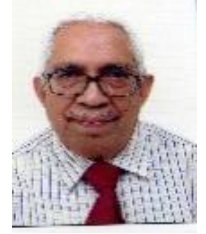

Prof. B. Dattaguru, retired as Chairman of Department of Aerospace Engineering, at Indian Institute of Science, Bangalore and later worked in Tech Mahindra and currently Professor at Jain (deemed-to-be-University). His fields of interest are Aerospace Structures and materials, Fracture and Computational mechanics. He graduated 26 Doctoral and 82 Master's students and published 207 Papers in Journals and conference proceedings. He received several awards, notable among them being Rustom Choksi award for excellence in Engineering Research (IISc), Academic Excellence award (DRDO) and Padmashree (Govt. of India), ICCES - 2014 Lifetime Achievement at Changwon, Korea and APCAM Senior Scientist awards at Sydney. 